\title{
Progressive Telangiectatic Rash
}

Alexandra Ritter, BS; Madison E. Hannay, DO; Joni Mazza-McCrann, MD; Jessica A. Forcucci, MD

This Dermatopathology Diagnosis in our print edition is eligible for 1 self-assessment credit for Maintenance of Certification from the American Board of Dermatology (ABD). After completing this activity, diplomates can visit the ABD website (http://www.abderm.org) to self-report the credits under the activity title "Cutis Dermatopathology Diagnosis." You may report the credit after each activity is completed or after accumulating multiple credits.

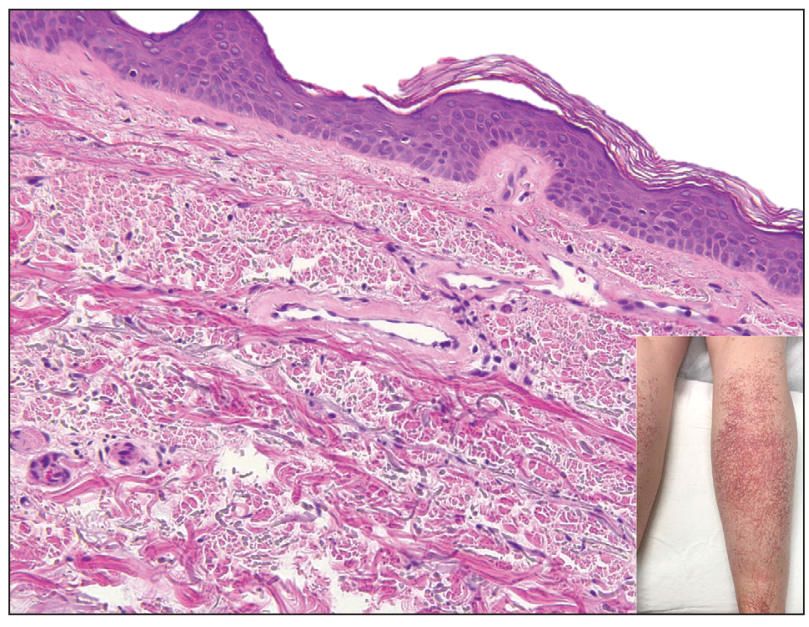

H\&E, original magnification $\times 200$ (clinical appearance of telangiectatic rash on the left leg [inset]).
A 54-year-old woman presented with purple-red vessels on the lower legs of 15 years' duration with gradual proximal progression to involve the thighs, breasts, and forearms. A punch biopsy of the inner thigh was obtained for histopathologic evaluation.

\section{THE BEST DIAGNOSIS IS:}
a. cutaneous collagenous vasculopathy
b. generalized essential telangiectasia
c. livedoid vasculopathy
d. primary systemic amyloidosis
e. stasis dermatitis

PLEASE TURN TO PAGE 125 FOR THE DIAGNOSIS

From the Medical University of South Carolina, Charleston. Drs. Hannay and Forcucci are from the Department of Pathology and Laboratory Medicine, and Dr. Mazza-McCrann is from the Department of Dermatology and Dermatologic Surgery.

The authors report no conflict of interest.

Correspondence: Madison E. Hannay, DO, 165 Ashley Ave, CH236E, Charleston, SC 29425 (hannay@musc.edu). doi:10.12788/cutis.0202 


\section{THE DIAGNOSIS:}

\section{Cutaneous Collagenous Vasculopathy}

${ }^{1}$ utaneous collagenous vasculopathy (CCV) is an idiopathic microangiopathy of the small vessels in the superficial dermis. A condition first identified by Salama and Rosenthal ${ }^{1}$ in 2000 , CCV likely is underreported, as its clinical mimickers are not routinely biopsied. ${ }^{2}$ It presents as asymptomatic telangiectatic macules, initially on the lower extremities and often spreading to the trunk. Cutaneous collagenous vasculopathy often is seen in middle-aged adults, and most patients have comorbidities such as hypertension, diabetes mellitus, or cardiovascular disease. The exact etiology of this disease is unknown. ${ }^{3,4}$

Histopathologically, CCV is characterized by dilated superficial vessels with thickened eosinophilic walls. The eosinophilic material is composed of hyalinized type IV collagen, which is periodic acid-Schiff positive and diastase resistant (Figure 1). ${ }^{3,4}$ Stains for amyloid are negative.

Generalized essential telangiectasia (GET) is a condition that presents with symmetric, blanchable, erythematous telangiectases. ${ }^{5}$ These lesions can occur alone or can accompany systemic diseases. Similar to CCV, the telangiectases tend to begin on the legs before gradually spreading to the trunk; however, this process more often is seen in females and occurs at an earlier age. Unlike CCV, GET can occur on mucosal surfaces, with cases of conjunctival and oral involvement reported. ${ }^{6}$ Generalized essential telangiectasia usually is a diagnosis of exclusion. ${ }^{7,8}$ It is thought that many CCV lesions have been misclassified clinically as GET, which highlights the importance of biopsy. Microscopically, GET is distinct from CCV in that the superficial dermis lacks thick-walled vessels. $^{5,7}$ Although usually not associated with systemic

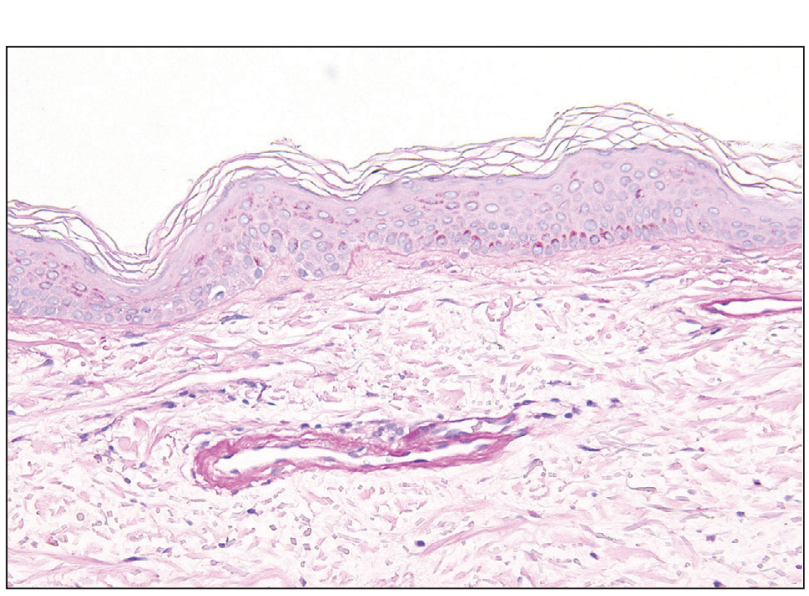

FIGURE 1. Cutaneous collagenous vasculopathy. Periodic acidSchiff staining demonstrated hyalinized vessel walls (original magnification $\times 200$ ) diseases or progressive morbidity, treatment options are limited. ${ }^{8}$

Livedoid vasculopathy, also known as atrophie blanche, is caused by fibrin thrombi occlusion of dermal vessels. Clinically, patients have recurrent telangiectatic papules and painful ulcers on the lower extremities that gradually heal, leaving behind white stellate scars. It is caused by an underlying prothrombotic state with a superimposed inflammatory response. ${ }^{9}$ Livedoid vasculopathy primarily affects middle-aged women, and many patients have comorbidities such as scleroderma or systemic lupus erythematosus. Histologically, the epidermis often is ulcerated, and thrombi are visualized within small vessels. Eosinophilic fibrinoid material is deposited in vessel walls, including but not confined to vessels at the base of the epidermal ulcer (Figure 2). The fibrinoid material is periodic acid-Schiff positive and diastase resistant and can be highlighted with immunofluorescence, which may help to distinguish this entity from CCV. ${ }^{1,9}$ As the disease progresses, vessels are diffusely hyalinized, and there is epidermal atrophy and dermal

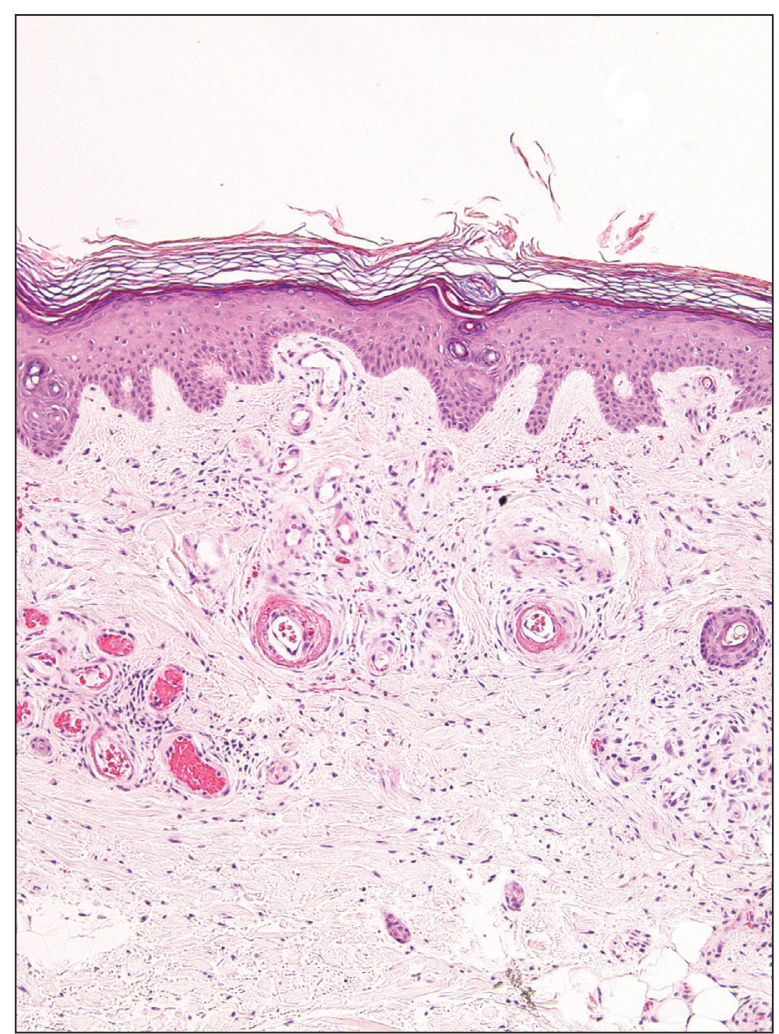

FIGURE 2. Livedoid vasculopathy (atrophie blanche). Fibrin thrombi within small vessels and vessel walls with adjacent stasis changes due to the anatomic site $(H \& E$, original magnification $\times 100)$. 

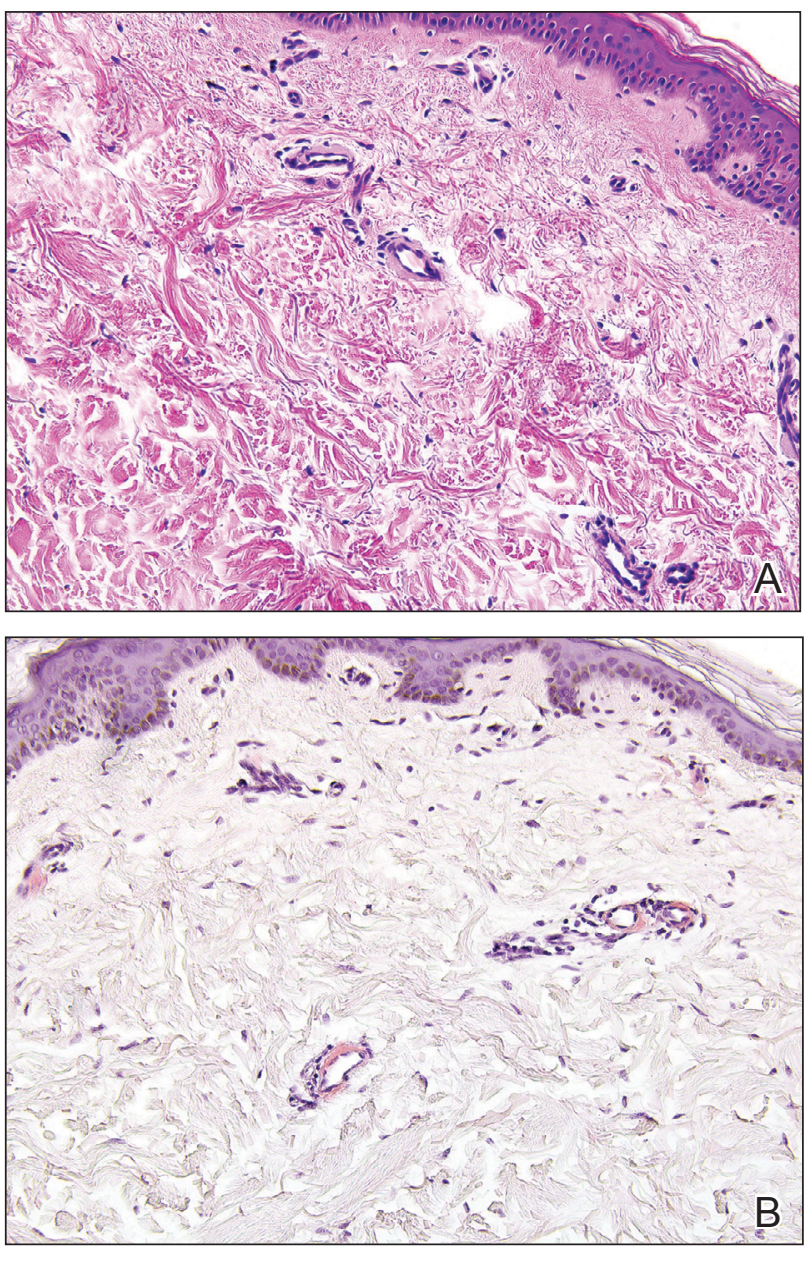

FIGURE 3. Amyloidosis. A, Amorphous eosinophilic material within the vessel walls $(H \& E$, original magnification $\times 200)$. B, Waxy redorange material involving vessel walls (Congo red, original magnification $\times 200$ ).

sclerosis. Treatment options include antiplatelet and fibrinolytic drugs with a multidisciplinary approach to resolve pain and scarring.

Primary systemic amyloidosis is a rare condition, and cutaneous manifestations are seen in approximately onethird of affected individuals. Amyloid deposition results in waxy papules that predominantly affect the face and periorbital areas but also may occur on the neck, flexural areas, and genitalia. ${ }^{5}$ Because the amyloid deposits also can be found within vessel walls, hemorrhagic lesions may occur. Microscopically, amorphous eosinophilic material can be found within the vessel walls, similar to CCV (Figure 3A); however, when stained with Congo red, cutaneous amyloidosis shows waxy red-orange material involving the vessel walls and exhibits apple green birefringence under polarization (Figure 3B).$^{10}$ Amyloid also will be negative for type IV collagen, fibronectin, and laminin, whereas $\mathrm{CCV}$ will be positive. ${ }^{5}$

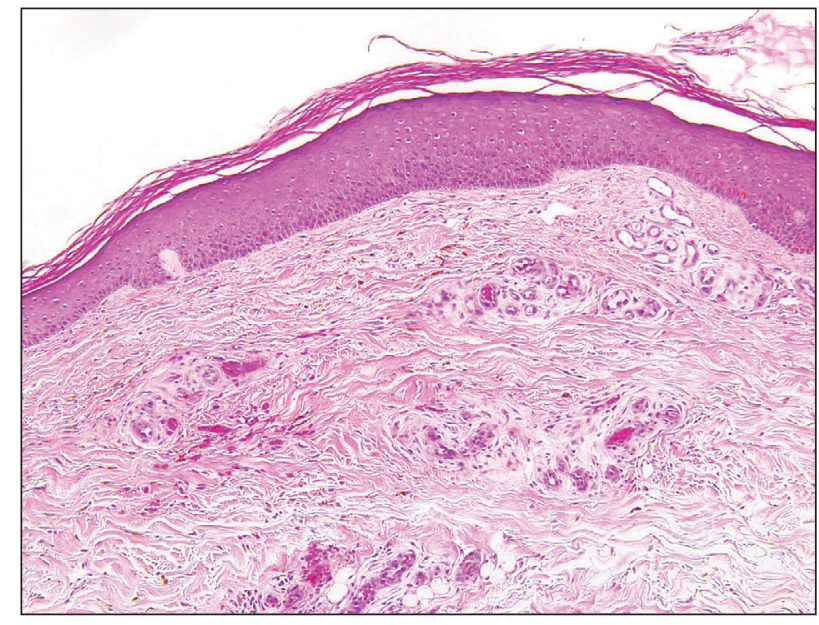

FIGURE 4. Stasis dermatitis. Thickened vessel walls with superficial dermal angioplasia, hemorrhage, and hemosiderin deposition $(\mathrm{H} \& \mathrm{E}$, original magnification $\times 100)$.

Stasis dermatitis is a result of chronic venous insufficiency and causes characteristic clinical and histopathologic findings. In contrast to $\mathrm{CCV}$, where hyalinized type IV collagen is deposited within the vessel wall, plasma and fibrin are deposited around the walls of capillaries in stasis dermatitis. ${ }^{11}$ Additional microscopic findings of stasis dermatitis include superficial dermal angioplasia, hemorrhage, and hemosiderin deposition (Figure 4).

\section{REFERENCES}

1. Salama S, Rosenthal D. Cutaneous collagenous vasculopathy with generalized telangiectasia: an immunohistochemical and ultrastructural study. J Cutan Pathol. 2000;27:40-48.

2. Bondier L, Tardieu M, Leveque P, et al. Cutaneous collagenous vasculopathy: report of two cases presenting as disseminated telangiectasias and review of the literature. Am J Dermatopathol. 2017;39:682-688.

3. Sartori DS, Almeida HL Jr, Dorn TV, et al. Cutaneous collagenous vasculopathy: light and transmission electron microscopy. An Bras Dermatol. 2019;94:211-213.

4. Brady BG, Ortleb M, Boyd AS, et al. Cutaneous collagenous vasculopa thy. J Clin Aesthet Dermatol. 2015;8:49-52.

5. Patterson JW, ed. Vascular tumors. Weedon's Skin Pathology. 4th ed. Churchill Livingstone/Elsevier; 2016:1069-1115.

6. Knöpfel N, Martín-Santiago A, Saus C, et al. Extensive acquired telangiectasias: comparison of generalized essential telangiectasia and cutaneous collagenous vasculopathy. Actas Dermosifiliogr. 2017;108:E21-E26.

7. Karimkhani C, Boyers LN, Olivere J, et al. Cutaneous collagenous vasculopathy. Cutis. 2019;103:E7-E8.

8. McGrae JD, Winkelmann RK. Generalized essential telangiectasia: report of a clinical and histochemical study of 13 patients with acquired cutaneous lesions. JAMA. 1963;185:909-913.

9. Vasudeva B, Neema S, Verma R. Livedoid vasculopathy: a review of pathogenesis and principles of management. Indian J Dermatol Venereol Leprol. 2016;82:478.

10. Ko CJ, Barr RJ. Color-pink. In: Ko CJ, Barr RJ, eds. Dermatopathology: Diagnosis by First Impression. 3rd ed. Wiley; 2016:303-322.

11. Clark ML, McGuinness AE, Vidal CI. Cutaneous collagenous vasculopathy: a unique case with positive direct immunofluorescence findings. Am J Dermatopathol. 2019;41:77-79. 